\title{
Mitochondria in Human Pluripotent Stem Cell
}

\section{Apoptosis}

Tara TeSlaa ${ }^{a}$, Kiyoko Setoguchi ${ }^{b}$, and Michael A. Teitell ${ }^{a, b, c,{ }^{*}}$

${ }^{a}$ Molecular Biology Institute, University of California, Los Angeles, CA, 90095, USA

${ }^{b}$ Department of Pathology and Laboratory Medicine, University of California, Los

Angeles, CA, 90095, USA

'Department of Bioengineering, Department of Pediatrics, California NanoSystems

Institute, Jonsson Comprehensive Cancer Center, and Broad Center of Regenerative

Medicine and Stem Cell Research, University of California, Los Angeles, CA, 90095,

USA

${ }^{*}$ Corresponding author: MA Teitell, Department of Pathology and Laboratory

Medicine, University of California, Los Angeles, 675 Charles E. Young Drive South, Los

Angeles, CA 90095, USA. Tel.: +1 310206 6754; Fax: +1 310267 0382; E-mail:

mteitell@mednet.ucla.edu 


\section{ABSTRACT}

Human pluripotent stem cells (hPSCs) have great potential in regenerative medicine because they can differentiate into any cell type in the body. Genome integrity is vital for human development and for high fidelity passage of genetic information across generations through the germ line. To ensure genome stability, hPSCs maintain a lower rate of mutation than somatic cells and undergo rapid apoptosis in response to DNA damage and additional cell stresses. Furthermore, cellular metabolism and the cell cycle are also differentially regulated between cells in pluripotent and differentiated states and can aid in protecting hPSCs against DNA damage and damaged cell propagation. Despite these safeguards, clinical use of hPSC derivatives could be compromised by tumorigenic potential and possible malignant transformation from failed to differentiate cells. Since hPSCs and mature cells differentially respond to cell stress, it may be possible to specifically target undifferentiated cells for rapid apoptosis in mixed cell populations to enable safer use of hPSC-differentiated cells in patients.

Keywords: apoptosis, human pluripotent stem cells, p53, tumorigenicity

Abbreviations: $\quad$ BAK, BCL-2 antagonist killer; BAX, BCL-2 associated $X$ protein; CDK1, cyclin dependent kinase 1; CLDN-6, claudin-6; CM, cardiomyocyte; CPE, Clostridium perfringens endotoxin; GPX2, glutathione peroxidase-2; GSH, glutathione; GSR, glutathione reductase; GSSG, glutathione disulfide; hESC, human embryonic stem cell; hiPSC, human induced pluripotent stem cell; hPSC, human pluripotent stem cell; IMS, intermembrane space; MDM2, mouse double minute 2 homolog; MMR, 
mismatch repair; MOMP, mitochondrial outer membrane permeabilization; NAMPT, nicotinamide phosphoribosyltransferase; OXPHOS, oxidative phosphorylation; PPP, pentose phosphate pathway; ROCK, Rho-associated kinase; ROS, reactive oxygen species; SCD1, stearoyl-coA desaturase; UCP2, uncoupling protein 2. 


\section{Introduction}

New possibilities in regenerative medicine have been enabled by the production of human embryonic stem cells (hESCs) and the reprogramming of somatic cells into human induced pluripotent stem cells (hiPSCs) [1-4]. Both cell types classify as human pluripotent stem cells (hPSCs) and each can self-renew indefinitely in culture. hPSCs also have the potential to develop into all of the mature cells in our bodies, including the germ cells that pass genetic information on to our progeny. Therefore, protection against harmful DNA mutations and other heritable lesions is vital for propagation of our species. Consistent with this fidelity, hPSCs exhibit a low rate of DNA mutation due to enhanced mechanisms of DNA damage protection and repair [5-9]. In the event that irreparable DNA or other forms of heritable damage do occur, however, suboptimal hPSCs are prevented from replicating by sensitive and rapid apoptotic mechanisms [1013]. hPSCs also uniquely regulate the cell cycle and cell cycle checkpoints to prevent damaged cells from further differentiation or cell division [14-16]. In this review we will examine and discuss the mechanisms employed by hPSCs to prevent the propagation of damaged cells, which centers on increased sensitivity to apoptosis triggers. Furthermore, teratoma formation in a rodent is the current gold standard test for hPSCs, but this occurrence also portends a potential tumorigenic threat for using hPSC derivative cells clinically [17]. An increased sensitivity to apoptotic stimuli for hPSCs could enable the selective removal of cells which fail to differentiate from a therapeutic cell population. Metabolic differences between hPSCs and their differentiated progeny are also substantial [18-23], potentially enabling metabolic selection against hPSCs in cell mixtures. Therefore, we also examine emerging methods for selective elimination of 
hPSCs that do not harm healthy populations of differentiated cells intended for cell therapies.

\section{2. hPSCs show increased sensitivity to apoptosis}

\subsection{Mitochondria and BCL-2 family proteins in apoptosis}

Apoptosis can be regulated by BCL-2 (B cell lymphoma-2) family proteins and their interactions with the mitochondrion. BCL-2 family proteins can be divided into three functional groups: pro-apoptotic effector proteins BAK (BCL-2 antagonist/killer) and BAX (BCL-2 associated $\mathrm{X}$ protein), a set of pro-apoptotic $\mathrm{BH}$ (BCL-2 homology domain 3)only proteins, and a set of pro-survival proteins that include BCL-2 itself [24]. BAK and BAX can initiate the apoptosis cascade by induction of mitochondrial outer membrane permeabilization (MOMP) $[25,26]$. BAK and BAX are activated through protein interactions that induce structural changes and their homo-oligomerization. Activation of BAX causes exposure of its $\mathrm{N}$-terminal domain and insertion of its $\mathrm{C}$-terminal domain into the mitochondrial outer membrane, which contrasts with BAK, which is localized to the mitochondrial outer membrane in both inactive and active conformations [27-29]. Homo-oligomerized BAK or BAX forms a pore that causes MOMP [25, 26, 30]. MOMP subsequently leads to the release of pro-apoptotic proteins in the mitochondrial intermembrane space (IMS), including cytochrome $c$, which activates caspase- 9 protease in the cytosol $[28,31,32]$. In turn, caspase- 9 activates additional effector caspases, unleashing a cascade of proteases that culminates in apoptotic cell death $[24,33,34]$. BAK and BAX have overlapping roles in apoptosis, but differ in their mechanisms of activation. Bak or Bax knockout mice have limited abnormalities, 
whereas a double knockout of both Bak and Bax is perinatal lethal due to defects in apoptosis induction [35]. In somatic cells, inactive BAX is located in the cytosol until an apoptotic stimulus causes BAX to interact with $\mathrm{p} 53$ or $\mathrm{BH} 3$-only proteins, activating $\mathrm{BAX}$ [27, 39-41]. BH3-only proteins can directly bind and activate BAK or BAX, or they can bind and neutralize pro-survival BCL-2 family member proteins. When pro-survival BCL2 proteins are not bound by BH3-only proteins, they can bind directly to activated BAK or BAX, inhibiting their pro-apoptotic activities [24]. Additional proteins can also interact with BCL-2 family member proteins to sensitize or deaden cellular responses to apoptosis induction [40,42-44]. Therefore, the cellular apoptotic threshold is a complex balance between pro-survival and pro-apoptotic proteins and their interactions.

\subsection{Differential roles of $B A K$ and $B A X$}

Some hESC lines show a unique pattern of BAX regulation during S-phase of the cell cycle, poising these lines for rapid apoptosis induction upon DNA damage. In these lines, during S-phase BAX is sequestered in the Golgi apparatus in its activated conformation, held away from the mitochondrion. Cell stress from DNA damage causes a rapid p53-dependent translocation of active BAX from the Golgi to the mitochondria by an unknown mechanism, causing apoptosis (Figure 1). By maintaining BAX in its active conformation in the Golgi, certain hESC lines bypass the BAX activation step and are 'primed' for a cell death response [11]. It is not yet known, however, how active BAX is localized to the Golgi and what holds it there prior to p53-dependent translocation to the mitochondrion. Active BAX forms homo-oligomers and pores activating MOMP [25] making it unclear whether active BAX also causes pore formation in the Golgi 
membrane stack and, if not, what prevents pore formation or oligomerization. Active BAX is detected by an antibody that recognizes its exposed N-terminal domain [11], but it is not known whether this antibody-detected conformational change is sufficient for BAX activity. The H1 hESC line lacks Golgi-localized active BAX but still exhibits hypersensitivity to apoptosis induction that is typical of other hPSC lines [10, 11, 45]. One interpretation of this data is that hPSC hypersensitivity to apoptosis may be independent of active BAX in the Golgi, or there may be additional mechanisms of hypersensitivity. In addition, whereas BAX has a key role in the apoptotic response of hPSCs to DNA damage, BAK is actually more important in response to other apoptotic insults and cell stresses. For example, the induction of rapid apoptosis in hPSCs by transcriptional inhibition using actinomycin D depends more on BAK than BAX [10]. Overall, some hPSC lines localize active BAX to the Golgi [11], but this unique localization is not required for apoptosis hypersensitivity in all hPSC lines, raising questions about its role in mitochondrial priming of hPSCs for apoptosis.

\subsection{Enhanced mitochondrial priming in hPSCs}

hPSCs also undergo rapid apoptosis with activation of the unfolded protein response and transcriptional inhibition $[10,12,13]$. These data strongly suggest that hPSCs are hypersensitive to multiple and perhaps all activators of mitochondrial-mediated apoptosis. The balance between pro- and anti-apoptotic BCL-2 family proteins controls the sensitivity of cells to mitochondrial-mediated apoptotic stimuli $[46,47]$. hPSCs are reportedly in a high mitochondrial priming state in which an elevated ratio of proapoptotic to pro-survival BCL-2 proteins are present in comparison to their differentiated 
progeny (Figure 1) [12]. However, the mechanistic underpinning for how mitochondrial priming is differentially regulated between hPSCs and differentiated cells remains unclear. Increased expression of pro-apoptotic BCL-2 family proteins occurs with the reprogramming of fibroblasts to hiPSCs, which could increase mitochondrial priming $[13,48]$. In addition, decreased expression of pro-survival BCL-2 itself has been reported in hESCs compared to differentiated cell types $[12,48]$. Finally, p53 can impact the activities of BCL-2 family proteins and MOMP, which is discussed next.

\section{P53, DNA damage, and cell cycle regulation}

\subsection{P53 in hPSCs}

The P53 tumor suppressor is activated by cell stress in both pluripotent and differentiated cells $[12,49]$. p53 is a key component of the DNA damage response through its roles as a transcription factor in the nucleus and through activation of BAK or BAX oligomerization at the mitochondria [40, 43, 44, 50-52]. The timing of induction of p53 target genes in response to DNA damage is similar in pluripotent and differentiated cells. When p53 is localized only to the cytoplasm, hPSCs can still undergo apoptosis induction without p53 transcriptional activity [12]. Therefore, both cytoplasmic and nuclear roles for p53 in apoptosis induction are functioning in hPSCs.

p53 protein stability is tightly regulated by the E3 ubiquitin ligase mouse double minute 2 homolog (MDM2), which triggers p53 degradation by mono-ubiquitination and degradation in the proteasome [53-55]. Disruption of a p53-MDM2 interaction in response to DNA damage is crucial for stabilizing p53 and its activities [53, 54]. In hPSCs, inhibition of MDM2 causes rapid p53 stabilization. Interestingly, inhibition of 
protein translation with cyclohexamide causes a fast proteasome-dependent decrease in p53 levels in hPSCs that does not occur in differentiated cells [10]. Therefore, p53 is unstable in hPSCs due to proteasome degradation. Additionally, deacetylation of p53, which promotes p53 degradation, is promoted by OCT4-dependent expression of SIRT1 [56]. Together the data suggest that hPSCs rapidly produce and degrade p53 in the absence of cellular stress, which enables quick p53 stabilization upon DNA damage or with other pro-apoptotic stimuli (Figure 1). Since p53 can directly regulate MOMP through interactions with BAK and BAX [40, 43, 44, 51], differences in p53 stability could account for differences in mitochondrial priming between hPSCs and differentiated cells. Contradicting this idea, mitochondrial priming in hPSCs was unaffected by p53 knockdown, suggesting that other mechanisms contribute to mitochondrial priming [12]. Overall, p53 has an early role in apoptosis for both hPSCs and differentiated cells, but differences in its turnover and stabilization rates increase apoptosis sensitivity for hPSCs with DNA damage.

\subsection{Heightened DNA damage protection and repair in $h P S C s$}

Compared to differentiated cells, hPSCs exhibit robust protection from DNA damage and heightened repair mechanisms $[5,8,13,58,59]$. For example, hPSCs have reduced DNA-damaging mitochondrial reactive oxygen species (ROS), with hiPSCs accumulating less nuclear and mitochondrial DNA damage when exposed to UV light or hydrogen peroxide compared to fibroblasts and most cancer cell lines $[13,60]$. Glutathione (GSH), a key intracellular antioxidant, is present at higher steady-state levels in hPSCs than fibroblasts, likely from increased expression of many antioxidant 
and detoxification enzymes (Figure 2) [13, 59, 60]. Glutathione peroxidase-2 (GPX2), which prevents oxidative damage by reducing free peroxides, is highly expressed in hPSCs. GPX2 generates glutathione disulfide (GSSG), which can be reduced back to antioxidant GSH by glutathione reductase (GSR), which is also highly expressed in hPSCs [13]. GSR, however, requires NADPH as a reducing agent cofactor for this reaction. Uncoupling protein 2 (UCP2), which is highly expressed in hPSCs, increases carbon flux through the pentose phosphate pathway (PPP), which produces NADPH (Figure 2) [18]. Nutrient metabolism also protects hPSCs from oxidative stress by favoring glycolysis over oxidative phosphorylation (OXPHOS) for ATP production, which results in lower ROS production from respiration [18, 60]. UCP2 appears well positioned to regulate these activities by reducing electron transport chain activity via transport of C-4 tricarboxylic acid cycle intermediate metabolites out of the mitochondria [18, 61]. Generally, hPSCs express DNA damage signaling and repair genes at higher levels than differentiated cells $[5,8,59]$. hiPSCs also have a more efficient DNA mismatch repair (MMR) pathway than fibroblasts due to higher expression of MMR proteins. Induction of a MMR-dependent response by alkylating DNA damage in hPSCs causes rapid p53 activation and apoptosis in the first cell cycle S-phase as contrasted with an arrest seen in the second cell cycle G2/M-phase after alkylating damage in somatic cell types [9]. Altogether, hPSCs limit DNA damage with reduced oxidative metabolism, high level antioxidant defense mechanisms, and robust DNA damage repair, but when these mechanisms fail, p53 is rapidly stabilized and hypersensitive apoptosis pathway activation is initiated. 


\subsection{Unique cell cycle regulation in hPSCs}

hPSCs show an abbreviated cell cycle due to a shortened G1-phase and they undergo p21-depedent cell cycle arrest in G2-phase with DNA damage [58, 62, 63]. As hPSCs differentiate, there is a concurrent p53-dependent increase in the duration of G1-phase [64]. Propagation of damaged hPSCs is also prevented by blocking differentiation during DNA replication and cell division, periods of the cell cycle with an increased probability for DNA damage. During G1-phase, hPSCs are most likely to differentiate, whereas maintained pluripotency is favored during replicative stress in S- and G2/Mphases of the cell cycle [14-16]. Interestingly, hPSCs selectively differentiate into endoderm during early G1-phase, whereas hPSCs preferentially differentiate into ectoderm during late G1-phase [16]. Promotion of pluripotency during DNA replication prevents cell fate changes and the propagation of cells with an increased probability of damaged DNA. Additionally, pluripotency maintenance during S-phase increases the probability of DNA damage repair and/or apoptosis, which are heightened responses in hPSCs.

\section{Dissociation induced apoptosis in hPSCs}

Single cell dissociation of hPSCs that normally grow in tightly packed colonies causes apoptosis, which can limit the use of techniques that require clonal isolation of hPSCs $[1,65]$. To overcome this limitation, inhibition of Rho-associated kinase (ROCK) increases both hPSC survival after dissociation and cloning efficiency [66]. Single cell dissociation of hPSCs disrupts E-cadherin-mediated intercellular contact, resulting in immediate membrane blebbing by ROCK hyperactivation of myosin $[67,68]$. Myosin 
activation contracts the actomyosin network and is required for dissociation-induced hPSC apoptosis [67-69]. Although dissociation-mediated apoptosis leads to MOMP and cytochrome $c$ release from mitochondria, the mechanism connecting actomyosin network contraction and MOMP initiation remains unknown [70].

\section{Selective apoptosis of hPSCs in therapeutic cell mixtures}

\subsection{Tumorigenicity of hPSCs}

A safety concern in developing hPSC derivatives for use in humans is their tumorigenic potential $[17,71]$. Teratoma formation as the main test for pluripotency illustrates the inherent tumorigenic potential of hPSCs [1, 2]. Furthermore, despite increased protection from DNA damage, extended in vitro culture of hPSCs somewhat paradoxically causes the accumulation of genetic lesions [72-74]. The most prevalent karyotypic change for hPSC lines is trisomy 12 , which increases $\mathrm{hPSC}$ proliferation rate and tumorigenic potential [72, 75-77]. In addition, hiPSCs can acquire genetic and epigenetic abnormalities during reprogramming to pluripotency $[17,78]$. A comparison of teratoma formation between hESCs and hiPSCs revealed that hiPSCs form teratomas more efficiently and more quickly than hESCs [79]. Therefore, therapeutic applications of hPSC differentiated derivatives will require increased control over innate tumorigenicity and possible malignant transformation of failed to differentiate cells.

In vitro differentiation to desired cell types remains inefficient, with some percentage of undifferentiated cells persisting within the population of differentiated cells to be transferred into patients [80]. Failure to differentiate is a common feature for cancers that maintain a high proliferative capacity and a potential sign of concern for malignant 
transformation [81-83]. Therefore, strategies to make differentiation close to $100 \%$ efficient, which currently seem unlikely, or to remove pluripotent residual cells while leaving healthy differentiated cells unharmed are of therapeutic interest. Current strategies include introduction of pluripotency-specific suicide genes [84-88], antibodybased enrichment or depletion of cell types [89, 90], lectin-targeted toxins [91], and selective induction of apoptosis by small molecule inhibitors that target hPSCs. Here, we examine small molecule inhibitors that may selectively remove hPSCs from heterogeneous cell populations. Many of these strategies take advantage of differences between hPSCs and differentiated cells, including apoptosis hypersensitivity and unique forms of cell metabolism.

\subsection{Exploiting hPSC hypersensitivity to apoptosis}

The balance of pro-apoptotic and pro-survival BCL-2 family proteins is different in pluripotent and differentiated cells, which makes strategies targeting this difference potentially useful for selective induction of apoptosis $[12,13]$. Since many cancers upregulate pro-survival BCL-2 family proteins, inhibitors targeting these proteins are in development and could find use in regenerative medicine [24]. Also, non-BCL-2 family pro-survival proteins, such as BCL-10 and SURVIVIN, are more highly expressed in hESCs than in adult stem/progenitor cells. Since adult stem/progenitor cells express other pro-survival proteins, such as BCL-2, chemical inhibition of BCL-10 or SURVIVIN causes apoptosis of hESCs, but not these more differentiated adult stem/progenitor cells. Hence, pretreatment of mixed populations of hESCs and differentiated cells with SURVIVIN inhibitors has been shown to repress teratoma formation in mice [57]. 
As discussed in Section 3.3, there is differential regulation of the cell cycle between hPSCs and differentiated cells. Systematic depletion of key cell-cycle regulatory proteins revealed that inhibition of cyclin dependent kinase 1 (CDK1) activates apoptosis in hPSCs but not in differentiated cells. Selective apoptosis of hPSCs by CDK1 inhibition is mediated by a p53-dependent increase in NOXA, a BH3-only proapoptotic protein [92].

As the hypersensitivity of hPSCs to apoptosis becomes better understood, additional strategies for removing hPSCs from differentiated cell mixtures will likely be identified. Since p53 may have a key role in hPSC apoptosis, MDM2 inhibitors may provide an interesting approach. Strategies that increase oxidative stress or DNA damage could provide selective cell death for hPSCs but at a price that seems unacceptable as the potential for increased DNA damage in differentiated cell types also increases.

\subsection{Metabolic selection against hPSCs}

hPSCs produce most ATP by glycolysis and transition to OXPHOS for most ATP production during differentiation [93, 94]. Cardiomyocytes (CMs), for example, produce most energy using glucose, fatty acids, and lactate by OXPHOS [95]. By culturing hPSCs and CMs in a mixture in conditions lacking glucose but supplemented with lactate, a population of CMs can be purified that lacks teratoma forming cells [95]. However, many other differentiated cell types cannot uptake and metabolize lactate making this strategy specific for CMs.

A small molecule inhibitor that can selectively kill hPSCs is STF-31. There are conflicting reports that STF-31 kills hPSCs by inhibiting either glucose transporters [96] 
or nicotinamide phosphoribosyltransferase (NAMPT) [97]. Facilitated glucose transporters are abundant at the hPSC cell membrane, which led to the identification of STF-31 [96]. The timing of STF-31-induced hPSC death, however, is distinct from the kinetics of hPSC death caused by WZB117, a glucose transporter-1 inhibitor. These discrepant temporal results leave open the possibility that STF-31 is acting through a different mechanism. An alternative potential mechanism is through inhibition of NAMPT, which is part of the NAD ${ }^{+}$salvage pathway. STF-31 treatment causes a decrease in $\mathrm{NAD}^{+}$levels before it causes a decrease in glucose uptake. Apoptosis of hPSCs by STF-31 treatment can be rescued by supplementation of nicotinic acid, supporting inhibition of NAMPT as a mechanism for STF-31 killing of hPSCs [97].

A small molecule inhibitor screen with readout for selective killing of hPSCs yielded an inhibitor of stearoyl-coA desaturase (SCD1), which catalyzes the conversion of saturated fatty acids to monounsaturated fatty acids [98]. Treatment of hPSCs with the SCD1 inhibitor, PluriSIn \#1, led to apoptosis after the induction of endoplasmic reticulum (ER) stress, which can be caused by saturated fatty acids [98-102]. PluriSIn \#1 treatment of a mixed population of pluripotent and differentiated cells for $48 \mathrm{~h}$ prevented teratoma formation in mice [98]. SCD1 in hPSCs may prevent cellular damage and oxidative stress because adding the saturated fatty acid palmitate can cause apoptosis, ER stress, mitochondrial ROS, and mitochondrial DNA damage [101, 103].

\subsection{Additional strategies for selective hPSC apoptosis}


Mitochondria in hPSCs appear granular and spherical with morphologically immature inner membrane cristae $[60,104,105]$. Whereas there are many characterized differences in metabolism between pluripotent and differentiated cells, very little is known about the differential regulation of mitochondrial biogenesis, dynamics, morphology, and function between these cell states. MitoBloCK-6, an inhibitor of the mitochondrial redox protein Erv1/ALR, is toxic to hPSCs but has no effect on differentiated cells [106]. Erv1/ALR cooperates with Mia40 to import proteins into the mitochondrial IMS via a disulfide relay system with cytochrome $c$ acting as the electron acceptor [107-109]. It remains unclear how hPSCs are specifically sensitive to MitoBloCK-6 treatment.

Tight junction protein claudin-6 (CLDN-6) is expressed specifically in hPSCs and not in differentiated cells. Although CLDN-6 is not required for hPSC survival and selfrenewal, it can be targeted with Clostridium perfringens enterotoxin (CPE), which binds to six claudin family member proteins, including CLDN-6, to induce cell death. CPE treatment inhibits teratoma formation of hPSCs in mice. However, CPE also targets CLDN-3, CLDN-4, CLDN-7, CLDN-8 and CLDN-14, which are expressed in various epithelial and endoderm cells. CPE utility, therefore, is limited to cell mixtures with differentiated tissue types that lack expression of these claudin family proteins [90].

\section{Concluding remarks}

hPSCs exhibit multiple molecular mechanisms for maintaining genome integrity and preventing cellular and organismal damage. However, many of the details for these mechanisms remain to be discovered. Mitochondria, which are central to the apoptotic 
process, are morphologically and functionally distinct between pluripotent and differentiated cells. Very little, however, is known about how these morphological and functional differences impact apoptosis sensitivity. Additionally, cellular metabolism, which is known to influence apoptosis sensitivity in somatic cells, also differs between the pluripotent and differentiated states [18-23, 110].

Pluripotent stem cells and cancer cells share many features including indefinite selfrenewal. Cancer cells, however, typically evolve mechanisms to avoid apoptosis despite significant DNA damage and other cell stresses [82]. By contrast, hPSCs are predisposed to apoptosis to maintain genome integrity. In many ways, including patterns of cell metabolism and proliferation, pluripotent stem cells and cancer cells resemble each other [111]. However, for programmed cell death, these cells seem to have diverged. Understanding how cell death mechanisms differ between cancer and pluripotent stem cells can potentially be leveraged to inform therapeutic developments for the benefit of both areas in human health and disease. 


\section{ACKNOWLEDGMENTS}

T.T. is supported by a Ruth L. Kirschstein National Research Service Award GM007185 and by the Broad Center of Regenerative Medicine and Stem Cell at UCLA Training Program. K.S. is supported by a California Institute for Regenerative Medicine (CIRM) Training Grant TG2-01169. M.A.T. is supported by CIRM Awards RB1-01397 and RT307678, by UC Discovery/NantWorks Biotechnology Award 178517, the Air Force Office of Scientific Research grant FA9550-15-1-0406, and by National Institutes of Health (NIH) Awards GM073981, CA156674, CA90571, GM114188, and CA185189. 


\section{FIGURE LEGENDS}

Fig 1. Mechanisms of hPSC hypersensitivity to apoptosis. hPSCs (top) and differentiated human cells (bottom) are shown before (left) and after (right) exposure to a cellular stress (yellow 'lightning bolt'). hPSCs initiate apoptosis (top right) in response to a stress, such as DNA damage, whereas differentiated cells may survive a similar stress (bottom right). Certain hPSC lines contain activated BAX (actBAX) located at the Golgi apparatus. DNA damage causes p53-dependent translocation of actBAX to the mitochondria to initiate MOMP and begin the apoptosis cascade. However, in differentiated cells BAX remains in the cytosol and is maintained in an inactive conformation (inBAX) through interactions with pro-survival proteins (green circles) and requires activation by interactions with $\mathrm{BH} 3-$ only pro-apoptotic proteins (red circles). hPSCs also show mitochondrial 'priming', indicating that the balance between proapoptotic and pro-survival mechanisms have been tipped to favor apoptosis compared to differentiated cells. Pro-apoptotic (red circles) and pro-survival (green circles) proteins depict mitochondrial 'priming' ratio in hPSCs (top left), in contrast to a more pro-survival protein ratio (bottom left) in differentiated cells. p53 is more rapidly degraded in hPSCs than in differentiated cells. Upon induction of apoptosis, hPSCs rapidly stabilize high concentrations of p53 which induces apoptosis through transcriptional and cytoplasmic/mitochondrial mechanisms of action.

Fig 2. Elevated expression of antioxidant enzymes drive robust GSH production in hPSCs. hPSCs express high levels of enzymes involved in GSH metabolism, including GPX2 and GSR. GPX2 catalyzes the reduction of hydrogen peroxide to water via the 
oxidation of GSH to GSSG. To refresh the GSH pool, GSR reduces GSSG to GSH through the oxidation of NADPH to NADP ${ }^{+}$. hPSCs have a high carbon flux through the PPP and low OXPHOS, which is at least partially due to high expression of UCP2 in hPSCs. The PPP produces NADPH which fuels glutathione metabolism. Low OXPHOS limits the ROS produced by the electron transport chain (ETC) of the mitochondrial inner membrane. 


\section{REFERENCES}

[1] Thomson JA, Itskovitz-Eldor J, Shapiro SS, Waknitz MA, Swiergiel JJ, Marshall VS, et al. Embryonic Stem Cell Lines Derived from Human Blastocysts. Science 1998;282:1145-7.

[2] Takahashi K, Tanabe K, Ohnuki M, Narita M, Ichisaka T, Tomoda K, et al. Induction of Pluripotent Stem Cells from Adult Human Fibroblasts by Defined Factors. Cell 2007;131:861-72.

[3] Lowry WE, Richter L, Yachechko R, Pyle AD, Tchieu J, Sridharan R, et al.

Generation of human induced pluripotent stem cells from dermal fibroblasts.

Proceedings of the National Academy of Sciences 2008;105:2883-8.

[4] Yu J, Vodyanik MA, Smuga-Otto K, Antosiewicz-Bourget J, Frane JL, Tian S, et al. Induced Pluripotent Stem Cell Lines Derived from Human Somatic Cells. Science 2007;318:1917-20.

[5] Momcilovic O, Knobloch L, Fornsaglio J, Varum S, Easley C, Schatten G. DNA Damage Responses in Human Induced Pluripotent Stem Cells and Embryonic Stem Cells. PLoS ONE 2010;5:e13410.

[6] Wilson KD, Sun N, Huang M, Zhang WY, Lee AS, Li Z, et al. Effects of lonizing Radiation on Self-Renewal and Pluripotency of Human Embryonic Stem Cells. Cancer Research 2010;70:5539-48.

[7] Cervantes RB, Stringer JR, Shao C, Tischfield JA, Stambrook PJ. Embryonic stem cells and somatic cells differ in mutation frequency and type. Proceedings of the National Academy of Sciences 2002;99:3586-90. 
[8] Maynard S, Swistowska AM, Lee JW, Liu Y, Liu S-T, Da Cruz AB, et al. Human Embryonic Stem Cells Have Enhanced Repair of Multiple Forms of DNA Damage. STEM CELLS 2008;26:2266-74.

[9] Lin B, Gupta D, Heinen CD. Human Pluripotent Stem Cells Have a Novel Mismatch Repair-dependent Damage Response. Journal of Biological Chemistry 2014;289:2431424.

[10] Setoguchi K, TeSlaa T, Koehler CM, Teitell MA. P53 Regulates Rapid Apoptosis in Human Pluripotent Stem Cells. Journal of Molecular Biology 2015.

[11] Dumitru R, Gama V, Fagan BM, Bower Jacquelyn J, Swahari V, Pevny Larysa H, et al. Human Embryonic Stem Cells Have Constitutively Active Bax at the Golgi and Are Primed to Undergo Rapid Apoptosis. Molecular Cell 2012;46:573-83.

[12] Liu Julia C, Guan X, Ryan Jeremy A, Rivera Ana G, Mock C, Agarwal V, et al. High Mitochondrial Priming Sensitizes hESCs to DNA-Damage-Induced Apoptosis. Cell Stem Cell 2013;13:483-91.

[13] Dannenmann B, Lehle S, Hildebrand Dominic G, Kübler A, Grondona P, Schmid V, et al. High Glutathione and Glutathione Peroxidase-2 Levels Mediate Cell-Type-Specific DNA Damage Protection in Human Induced Pluripotent Stem Cells. Stem Cell Reports 2015;4:886-98.

[14] Sela Y, Molotski N, Golan S, Itskovitz-Eldor J, Soen Y. Human Embryonic Stem Cells Exhibit Increased Propensity to Differentiate During the G1 Phase Prior to Phosphorylation of Retinoblastoma Protein. STEM CELLS 2012;30:1097-108. 
[15] Gonzales Kevin Andrew U, Liang H, Lim Y-S, Chan Y-S, Yeo J-C, Tan C-P, et al. Deterministic Restriction on Pluripotent State Dissolution by Cell-Cycle Pathways. Cell 2015;162:564-79.

[16] Pauklin S, Vallier L. The Cell-Cycle State of Stem Cells Determines Cell Fate Propensity. Cell 2013;155:135-47.

[17] Ben-David U, Benvenisty N. The tumorigenicity of human embryonic and induced pluripotent stem cells. Nat Rev Cancer 2011;11:268-77.

[18] Zhang J, Khvorostov I, Hong JS, Oktay Y, Vergnes L, Nuebel E, et al. UCP2 regulates energy metabolism and differentiation potential of human pluripotent stem cells. The EMBO Journal 2011;30:4860-73.

[19] Zhou W, Choi M, Margineantu D, Margaretha L, Hesson J, Cavanaugh C, et al. HIF1a induced switch from bivalent to exclusively glycolytic metabolism during ESC to - EpiSC/hESC transition. The EMBO journal 2012;31:2103-16.

[20] Varum S, Rodrigues AS, Moura MB, Momcilovic O, Easley CAIV, Ramalho-Santos J, et al. Energy Metabolism in Human Pluripotent Stem Cells and Their Differentiated Counterparts. PLoS ONE 2011;6:e20914.

[21] Moussaieff A, Rouleau M, Kitsberg D, Cohen M, Levy G, Barasch D, et al. Glycolysis-Mediated Changes in Acetyl-CoA and Histone Acetylation Control the Early Differentiation of Embryonic Stem Cells. Cell Metabolism 2015;21:392-402.

[22] Zhang J, Nuebel E, Daley George Q, Koehler Carla M, Teitell Michael A. Metabolic Regulation in Pluripotent Stem Cells during Reprogramming and Self-Renewal. Cell Stem Cell 2012;11:589-95. 
[23] Panopoulos AD, Yanes O, Ruiz S, Kida YS, Diep D, Tautenhahn R, et al. The metabolome of induced pluripotent stem cells reveals metabolic changes occurring in somatic cell reprogramming. Cell Res 2012;22:168-77.

[24] Czabotar PE, Lessene G, Strasser A, Adams JM. Control of apoptosis by the BCL2 protein family: implications for physiology and therapy. Nat Rev Mol Cell Biol 2014;15:49-63.

[25] Czabotar Peter E, Westphal D, Dewson G, Ma S, Hockings C, Fairlie WD, et al. Bax Crystal Structures Reveal How BH3 Domains Activate Bax and Nucleate Its Oligomerization to Induce Apoptosis. Cell 2013;152:519-31.

[26] Kim H, Tu H-C, Ren D, Takeuchi O, Jeffers JR, Zambetti GP, et al. Stepwise Activation of BAX and BAK by tBID, BIM, and PUMA Initiates Mitochondrial Apoptosis. Molecular Cell 2009;36:487-99.

[27] Gavathiotis E, Reyna DE, Davis ML, Bird GH, Walensky LD. BH3-Triggered Structural Reorganization Drives the Activation of Proapoptotic BAX. Molecular Cell 2010;40:481-92.

[28] Wei MC, Lindsten T, Mootha VK, Weiler S, Gross A, Ashiya M, et al. tBID, a membrane-targeted death ligand, oligomerizes BAK to release cytochrome c. Genes \& Development 2000;14:2060-71.

[29] Griffiths GJ, Dubrez L, Morgan CP, Jones NA, Whitehouse J, Corfe BM, et al. Cell Damage-induced Conformational Changes of the Pro-Apoptotic Protein Bak In Vivo Precede the Onset of Apoptosis. The Journal of Cell Biology 1999;144:903-14. 
[30] Dai H, Smith A, Meng XW, Schneider PA, Pang Y-P, Kaufmann SH. Transient binding of an activator $\mathrm{BH} 3$ domain to the Bak $\mathrm{BH} 3$-binding groove initiates Bak oligomerization. The Journal of Cell Biology 2011;194:39-48.

[31] Desagher S, Osen-Sand A, Nichols A, Eskes R, Montessuit S, Lauper S, et al. Bidinduced Conformational Change of Bax Is Responsible for Mitochondrial Cytochrome c Release during Apoptosis. The Journal of Cell Biology 1999;144:891-901.

[32] Jürgensmeier JM, Xie Z, Deveraux Q, Ellerby L, Bredesen D, Reed JC. Bax directly induces release of cytochrome $\mathrm{c}$ from isolated mitochondria. Proceedings of the National Academy of Sciences 1998;95:4997-5002.

[33] Zou H, Henzel WJ, Liu X, Lutschg A, Wang X. Apaf-1, a Human Protein Homologous to C. elegans CED-4, Participates in Cytochrome c-Dependent Activation of Caspase-3. Cell 1997;90:405-13.

[34] Li P, Nijhawan D, Budihardjo I, Srinivasula SM, Ahmad M, Alnemri ES, et al. Cytochrome $c$ and dATP-Dependent Formation of Apaf-1/Caspase-9 Complex Initiates an Apoptotic Protease Cascade. Cell 1997;91:479-89.

[35] Lindsten T, Ross AJ, King A, Zong W-X, Rathmell JC, Shiels HA, et al. The Combined Functions of Proapoptotic Bcl-2 Family Members Bak and Bax Are Essential for Normal Development of Multiple Tissues. Molecular Cell 2000;6:1389-99.

[36] Leshchiner ES, Braun CR, Bird GH, Walensky LD. Direct activation of full-length proapoptotic BAK. Proceedings of the National Academy of Sciences 2013;110:E986E95. 
[37] Moldoveanu T, Grace CR, Llambi F, Nourse A, Fitzgerald P, Gehring K, et al. BIDinduced structural changes in BAK promote apoptosis. Nat Struct Mol Biol 2013;20:58997.

[38] Vela L, Gonzalo O, Naval J, Marzo I. Direct Interaction of Bax and Bak Proteins with Bcl-2 Homology Domain 3 (BH3)-only Proteins in Living Cells Revealed by Fluorescence Complementation. Journal of Biological Chemistry 2013;288:4935-46. [39] Walensky LD, Pitter K, Morash J, Oh KJ, Barbuto S, Fisher J, et al. A Stapled BID BH3 Helix Directly Binds and Activates BAX. Molecular Cell 2006;24:199-210. [40] Chipuk JE, Kuwana T, Bouchier-Hayes L, Droin NM, Newmeyer DD, Schuler M, et al. Direct Activation of Bax by p53 Mediates Mitochondrial Membrane Permeabilization and Apoptosis. Science 2004;303:1010-4.

[41] Gavathiotis E, Suzuki M, Davis ML, Pitter K, Bird GH, Katz SG, et al. BAX activation is initiated at a novel interaction site. Nature 2008;455:1076-81. [42] Vousden KH, Prives C. Blinded by the Light: The Growing Complexity of p53. Cell 2009;137:413-31.

[43] Leu JIJ, Dumont P, Hafey M, Murphy ME, George DL. Mitochondrial p53 activates Bak and causes disruption of a Bak-Mcl1 complex. Nat Cell Biol 2004;6:443-50. [44] Pietsch EC, Perchiniak E, Canutescu AA, Wang G, Dunbrack RL, Murphy ME. Oligomerization of BAK by p53 Utilizes Conserved Residues of the p53 DNA Binding Domain. Journal of Biological Chemistry 2008;283:21294-304.

[45] Liu JC, Lerou PH, Lahav G. Stem cells: balancing resistance and sensitivity to DNA damage. Trends in Cell Biology 2014;24:268-74. 
[46] Certo M, Moore VDG, Nishino M, Wei G, Korsmeyer S, Armstrong SA, et al.

Mitochondria primed by death signals determine cellular addiction to antiapoptotic BCL2 family members. Cancer Cell 2006;9:351-65.

[47] Chonghaile TN, Sarosiek KA, Vo T-T, Ryan JA, Tammareddi A, Moore VDG, et al. Pretreatment Mitochondrial Priming Correlates with Clinical Response to Cytotoxic Chemotherapy. Science 2011;334:1129-33.

[48] Madden DT, Davila-Kruger D, Melov S, Bredesen DE. Human Embryonic Stem Cells Express Elevated Levels of Multiple Pro-Apoptotic BCL-2 Family Members. PLoS ONE 2011;6:e28530.

[49] Grandela C, Pera MF, Wolvetang EJ. p53 is required for etoposide-induced apoptosis of human embryonic stem cells. Stem Cell Research 2008;1:116-28.

[50] Green DR, Kroemer G. Cytoplasmic functions of the tumour suppressor p53. Nature 2009;458:1127-30.

[51] Mihara M, Erster S, Zaika A, Petrenko O, Chittenden T, Pancoska P, et al. p53 Has a Direct Apoptogenic Role at the Mitochondria. Molecular Cell 2003;11:577-90. [52] Vousden KH, Lu X. Live or let die: the cell's response to p53. Nat Rev Cancer 2002;2:594-604.

[53] Haupt Y, Maya R, Kazaz A, Oren M. Mdm2 promotes the rapid degradation of p53. Nature 1997;387:296-9.

[54] Kubbutat MHG, Jones SN, Vousden KH. Regulation of p53 stability by Mdm2. Nature 1997;387:299-303. 
[55] Momand J, Zambetti GP, Olson DC, George D, Levine AJ. The mdm-2 oncogene product forms a complex with the p53 protein and inhibits p53-mediated transactivation. Cell 1992;69:1237-45.

[56] Zhang Z-N, Chung S-K, Xu Z, Xu Y. Oct4 Maintains the Pluripotency of Human Embryonic Stem Cells by Inactivating p53 Through Sirt1-Mediated Deacetylation. STEM CELLS 2014;32:157-65.

[57] Lee M-O, Moon SH, Jeong H-C, Yi J-Y, Lee T-H, Shim SH, et al. Inhibition of pluripotent stem cell-derived teratoma formation by small molecules. Proceedings of the National Academy of Sciences 2013;110:E3281-E90.

[58] Filion TM, Qiao M, Ghule PN, Mandeville M, van Wijnen AJ, Stein JL, et al. Survival responses of human embryonic stem cells to DNA damage. Journal of Cellular Physiology 2009;220:586-92.

[59] Saretzki G, Walter T, Atkinson S, Passos JF, Bareth B, Keith WN, et al. Downregulation of Multiple Stress Defense Mechanisms During Differentiation of Human Embryonic Stem Cells. STEM CELLS 2008;26:455-64.

[60] Prigione A, Fauler B, Lurz R, Lehrach H, Adjaye J. The Senescence-Related Mitochondrial/Oxidative Stress Pathway is Repressed in Human Induced Pluripotent Stem Cells. STEM CELLS 2010;28:721-33.

[61] Vozza A, Parisi G, De Leonardis F, Lasorsa FM, Castegna A, Amorese D, et al. UCP2 transports C4 metabolites out of mitochondria, regulating glucose and glutamine oxidation. Proceedings of the National Academy of Sciences 2014;111:960-5. 
[62] Becker KA, Ghule PN, Therrien JA, Lian JB, Stein JL, van Wijnen AJ, et al. Selfrenewal of human embryonic stem cells is supported by a shortened $\mathrm{G} 1$ cell cycle phase. Journal of Cellular Physiology 2006;209:883-93.

[63] Momčilović O, Choi S, Varum S, Bakkenist C, Schatten G, Navara C. Ionizing Radiation Induces Ataxia Telangiectasia Mutated-Dependent Checkpoint Signaling and G2 But Not G1 Cell Cycle Arrest in Pluripotent Human Embryonic Stem Cells. STEM CELLS 2009;27:1822-35.

[64] Jain AK, Allton K, lacovino M, Mahen E, Milczarek RJ, Zwaka TP, et al. p53 Regulates Cell Cycle and MicroRNAs to Promote Differentiation of Human Embryonic Stem Cells. PLoS Biol 2012;10:e1001268.

[65] Reubinoff BE, Pera MF, Fong C-Y, Trounson A, Bongso A. Embryonic stem cell lines from human blastocysts: somatic differentiation in vitro. Nat Biotech 2000;18:399404.

[66] Watanabe K, Ueno M, Kamiya D, Nishiyama A, Matsumura M, Wataya T, et al. A ROCK inhibitor permits survival of dissociated human embryonic stem cells. Nat Biotech 2007;25:681-6.

[67] Ohgushi M, Matsumura M, Eiraku M, Murakami K, Aramaki T, Nishiyama A, et al. Molecular Pathway and Cell State Responsible for Dissociation-Induced Apoptosis in Human Pluripotent Stem Cells. Cell Stem Cell 2010;7:225-39.

[68] Chen G, Hou Z, Gulbranson DR, Thomson JA. Actin-Myosin Contractility Is Responsible for the Reduced Viability of Dissociated Human Embryonic Stem Cells. Cell Stem Cell 2010;7:240-8. 
[69] Charras G, Paluch E. Blebs lead the way: how to migrate without lamellipodia. Nat Rev Mol Cell Biol 2008;9:730-6.

[70] Samuel MS, Olson MF. Dying Alone: A Tale of Rho. Cell Stem Cell 2010;7:135-6.

[71] Lee AS, Tang C, Rao MS, Weissman IL, Wu JC. Tumorigenicity as a clinical hurdle for pluripotent stem cell therapies. Nat Med 2013;19:998-1004.

[72] Draper JS, Smith K, Gokhale P, Moore HD, Maltby E, Johnson J, et al. Recurrent gain of chromosomes $17 q$ and 12 in cultured human embryonic stem cells. Nat Biotech 2004;22:53-4.

[73] Cowan CA, Klimanskaya I, McMahon J, Atienza J, Witmyer J, Zucker JP, et al. Derivation of Embryonic Stem-Cell Lines from Human Blastocysts. New England Journal of Medicine 2004;350:1353-6.

[74] Taapken SM, Nisler BS, Newton MA, Sampsell-Barron TL, Leonhard KA, McIntire EM, et al. Karyotypic abnormalities in human induced pluripotent stem cells and embryonic stem cells. Nat Biotech 2011;29:313-4.

[75] Baker DEC, Harrison NJ, Maltby E, Smith K, Moore HD, Shaw PJ, et al. Adaptation to culture of human embryonic stem cells and oncogenesis in vivo. Nat Biotech 2007;25:207-15.

[76] Mayshar Y, Ben-David U, Lavon N, Biancotti J-C, Yakir B, Clark AT, et al. Identification and Classification of Chromosomal Aberrations in Human Induced Pluripotent Stem Cells. Cell Stem Cell 2010;7:521-31.

[77] Ben-David U, Arad G, Weissbein U, Mandefro B, Maimon A, Golan-Lev T, et al. Aneuploidy induces profound changes in gene expression, proliferation and tumorigenicity of human pluripotent stem cells. Nat Commun 2014;5. 
[78] Lister R, Pelizzola M, Kida YS, Hawkins RD, Nery JR, Hon G, et al. Hotspots of aberrant epigenomic reprogramming in human induced pluripotent stem cells. Nature $2011 ; 471: 68-73$.

[79] Gutierrez-Aranda I, Ramos-Mejia V, Bueno C, Munoz-Lopez M, Real PJ, Mácia A, et al. Human Induced Pluripotent Stem Cells Develop Teratoma More Efficiently and Faster Than Human Embryonic Stem Cells Regardless the Site of Injection. STEM CELLS 2010;28:1568-70.

[80] Miura K, Okada Y, Aoi T, Okada A, Takahashi K, Okita K, et al. Variation in the safety of induced pluripotent stem cell lines. Nat Biotech 2009;27:743-5.

[81] Tenen DG. Disruption of differentiation in human cancer: AML shows the way. Nat Rev Cancer 2003;3:89-101.

[82] Hanahan D, Weinberg RA. The Hallmarks of Cancer. Cell 2000;100:57-70. [83] Lu C, Ward PS, Kapoor GS, Rohle D, Turcan S, Abdel-Wahab O, et al. IDH mutation impairs histone demethylation and results in a block to cell differentiation. Nature 2012;483:474-8.

[84] Schuldiner M, Itskovitz-Eldor J, Benvenisty N. Selective Ablation of Human Embryonic Stem Cells Expressing a "Suicide" Gene. STEM CELLS 2003;21:257-65. [85] Anderson D, Self T, Mellor IR, Goh G, Hill SJ, Denning C. Transgenic Enrichment of Cardiomyocytes From Human Embryonic Stem Cells. Mol Ther 2007;15:2027-36. [86] Rong Z, Fu X, Wang M, Xu Y. A Scalable Approach to Prevent Teratoma Formation of Human Embryonic Stem Cells. Journal of Biological Chemistry 2012;287:32338-45. 
[87] Cheng F, Ke Q, Chen F, Cai B, Gao Y, Ye C, et al. Protecting against wayward human induced pluripotent stem cells with a suicide gene. Biomaterials 2012;33:3195204.

[88] Yagyu S, Hoyos V, Del Bufalo F, Brenner MK. An Inducible Caspase-9 Suicide Gene to Improve the Safety of Therapy Using Human Induced Pluripotent Stem Cells. Mol Ther 2015;23:1475-85.

[89] Tang C, Lee AS, Volkmer J-P, Sahoo D, Nag D, Mosley AR, et al. An antibody against SSEA-5 glycan on human pluripotent stem cells enables removal of teratomaforming cells. Nat Biotech 2011;29:829-34.

[90] Ben-David U, Nudel N, Benvenisty N. Immunologic and chemical targeting of the tight-junction protein Claudin-6 eliminates tumorigenic human pluripotent stem cells. Nat Commun 2013;4.

[91] Tateno H, Onuma $Y$, Ito $Y$, Minoshima F, Saito S, Shimizu M, et al. Elimination of Tumorigenic Human Pluripotent Stem Cells by a Recombinant Lectin-Toxin Fusion Protein. Stem Cell Reports 2015;4:811-20.

[92] Huskey Noelle E, Guo T, Evason Kimberley J, Momcilovic O, Pardo D, Creasman Katelyn J, et al. CDK1 Inhibition Targets the p53-NOXA-MCL1 Axis, Selectively Kills Embryonic Stem Cells, and Prevents Teratoma Formation. Stem Cell Reports 2015. [93] Zhang J, Khvorostov I, Hong JS, Oktay Y, Vergnes L, Nuebel E, et al. UCP2 regulates energy metabolism and differentiation potential of human pluripotent stem cells. The EMBO journal 2011;30:4860-73. 
[94] Zhang J, Nuebel E, Daley George Q, Koehler Carla M, Teitell Michael A. Metabolic Regulation in Pluripotent Stem Cells during Reprogramming and Self-Renewal. Cell Stem Cell;11:589-95.

[95] Tohyama S, Hattori F, Sano M, Hishiki T, Nagahata Y, Matsuura T, et al. Distinct Metabolic Flow Enables Large-Scale Purification of Mouse and Human Pluripotent Stem Cell-Derived Cardiomyocytes. Cell Stem Cell 2013;12:127-37.

[96] Boheler Kenneth R, Bhattacharya S, Kropp Erin M, Chuppa S, Riordon Daniel R, Bausch-Fluck D, et al. A Human Pluripotent Stem Cell Surface N-Glycoproteome Resource Reveals Markers, Extracellular Epitopes, and Drug Targets. Stem Cell Reports 2014;3:185-203.

[97] Kropp EM, Oleson BJ, Broniowska KA, Bhattacharya S, Chadwick AC, Diers AR, et al. Inhibition of an NAD+ Salvage Pathway Provides Efficient and Selective Toxicity to Human Pluripotent Stem Cells. Stem Cells Translational Medicine 2015.

[98] Ben-David U, Gan Q-F, Golan-Lev T, Arora P, Yanuka O, Oren Yifat S, et al. Selective Elimination of Human Pluripotent Stem Cells by an Oleate Synthesis Inhibitor Discovered in a High-Throughput Screen. Cell Stem Cell 2013;12:167-79. [99] Wei Y, Wang D, Topczewski F, Pagliassotti MJ. Saturated fatty acids induce endoplasmic reticulum stress and apoptosis independently of ceramide in liver cells. American Journal of Physiology - Endocrinology and Metabolism 2006;291:E275-E81. [100] Wang D, Wei Y, Pagliassotti MJ. Saturated Fatty Acids Promote Endoplasmic Reticulum Stress and Liver Injury in Rats with Hepatic Steatosis. Endocrinology 2006;147:943-51. 
[101] Yuzefovych L, Wilson G, Rachek L. Different effects of oleate vs. palmitate on mitochondrial function, apoptosis, and insulin signaling in L6 skeletal muscle cells: role of oxidative stress. American Journal of Physiology - Endocrinology and Metabolism 2010;299:E1096-E105.

[102] Karaskov E, Scott C, Zhang L, Teodoro T, Ravazzola M, Volchuk A. Chronic Palmitate But Not Oleate Exposure Induces Endoplasmic Reticulum Stress, Which May Contribute to INS-1 Pancreatic $\beta$-Cell Apoptosis. Endocrinology 2006;147:3398-407. [103] Listenberger LL, Ory DS, Schaffer JE. Palmitate-induced Apoptosis Can Occur through a Ceramide-independent Pathway. Journal of Biological Chemistry $2001 ; 276: 14890-5$.

[104] St John J, Amaral A, Bowles E, Oliveira J, Lloyd R, Freitas M, et al. The Analysis of Mitochondria and Mitochondrial DNA in Human Embryonic Stem Cells. In: Turksen K, editor. Human Embryonic Stem Cell Protocols: Humana Press; 2006. p. 347-74. [105] Suhr ST, Chang EA, Tjong J, Alcasid N, Perkins GA, Goissis MD, et al. Mitochondrial Rejuvenation After Induced Pluripotency. PLoS ONE 2010;5:e14095. [106] Dabir Deepa V, Hasson Samuel A, Setoguchi K, Johnson Meghan E, Wongkongkathep P, Douglas Colin J, et al. A Small Molecule Inhibitor of RedoxRegulated Protein Translocation into Mitochondria. Developmental Cell 2013;25:81-92. [107] Mesecke N, Terziyska N, Kozany C, Baumann F, Neupert W, Hell K, et al. A Disulfide Relay System in the Intermembrane Space of Mitochondria that Mediates Protein Import. Cell 2005;121:1059-69. 
[108] Dabir DV, Leverich EP, Kim SK, Tsai FD, Hirasawa M, Knaff DB, et al. A role for cytochrome $\mathrm{c}$ and cytochrome $\mathrm{c}$ peroxidase in electron shuttling from Erv1. The EMBO Journal 2007;26:4801-11.

[109] Allen S, Balabanidou V, Sideris DP, Lisowsky T, Tokatlidis K. Erv1 Mediates the Mia40-dependent Protein Import Pathway and Provides a Functional Link to the Respiratory Chain by Shuttling Electrons to Cytochrome c. Journal of Molecular Biology 2005;353:937-44.

[110] Green DR, Galluzzi L, Kroemer G. Metabolic control of cell death. Science $2014 ; 345$.

[111] Hanahan D, Weinberg RA. Hallmarks of cancer: the next generation. cell 2011;144:646-74. 
$\bigodot$ pro-survival protein

\section{( pro-apoptosis protein}
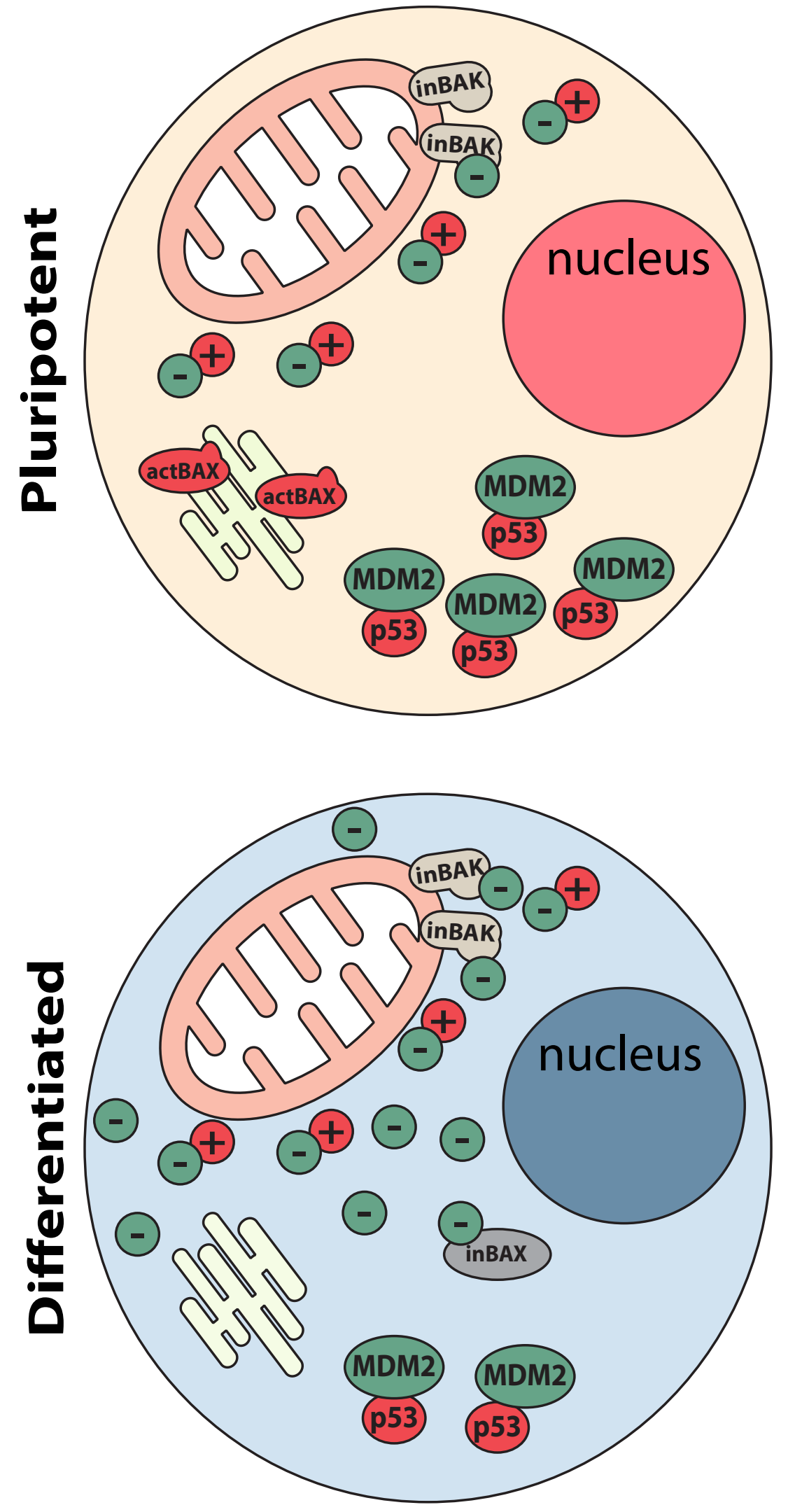

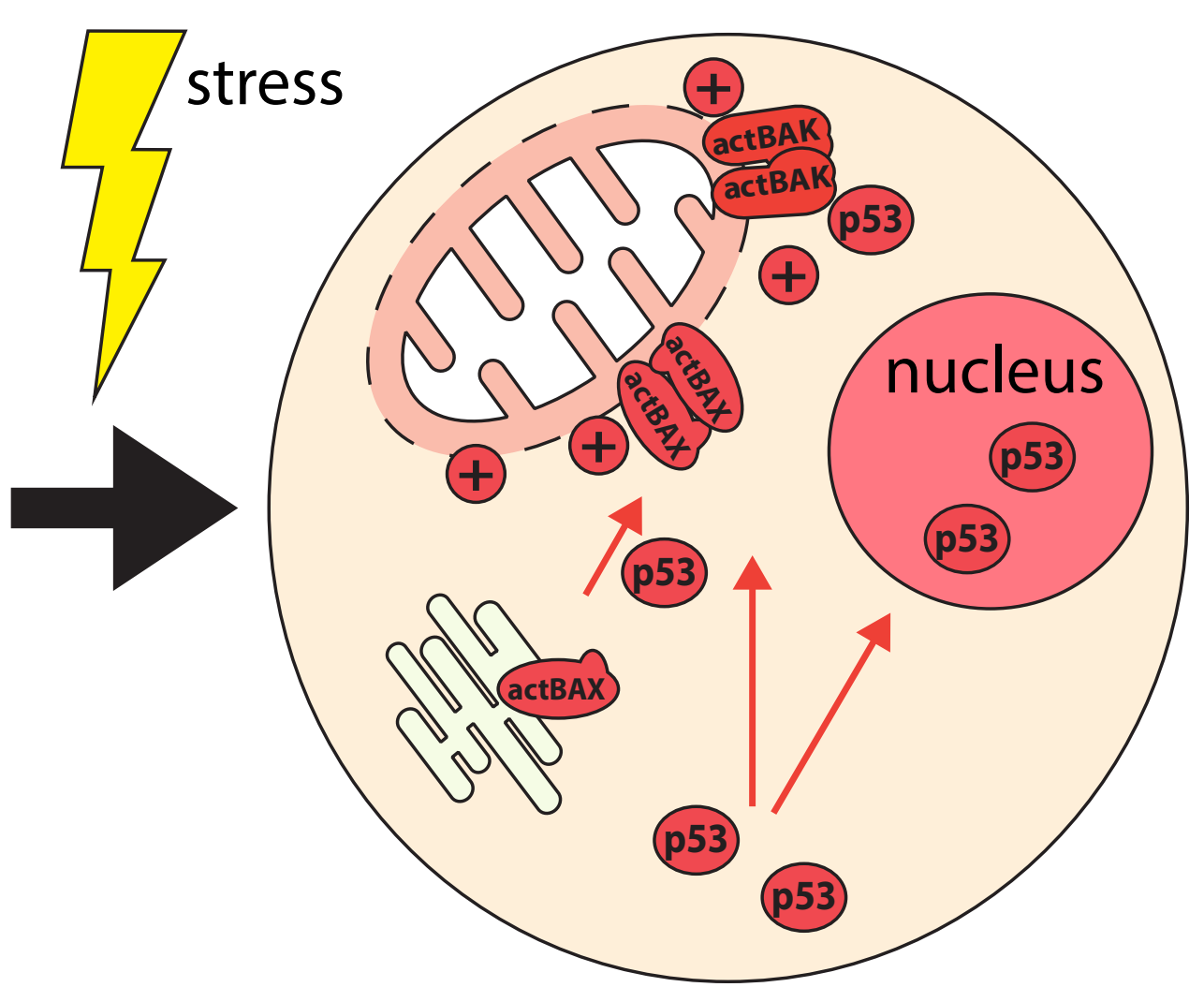

\section{Apoptosis}

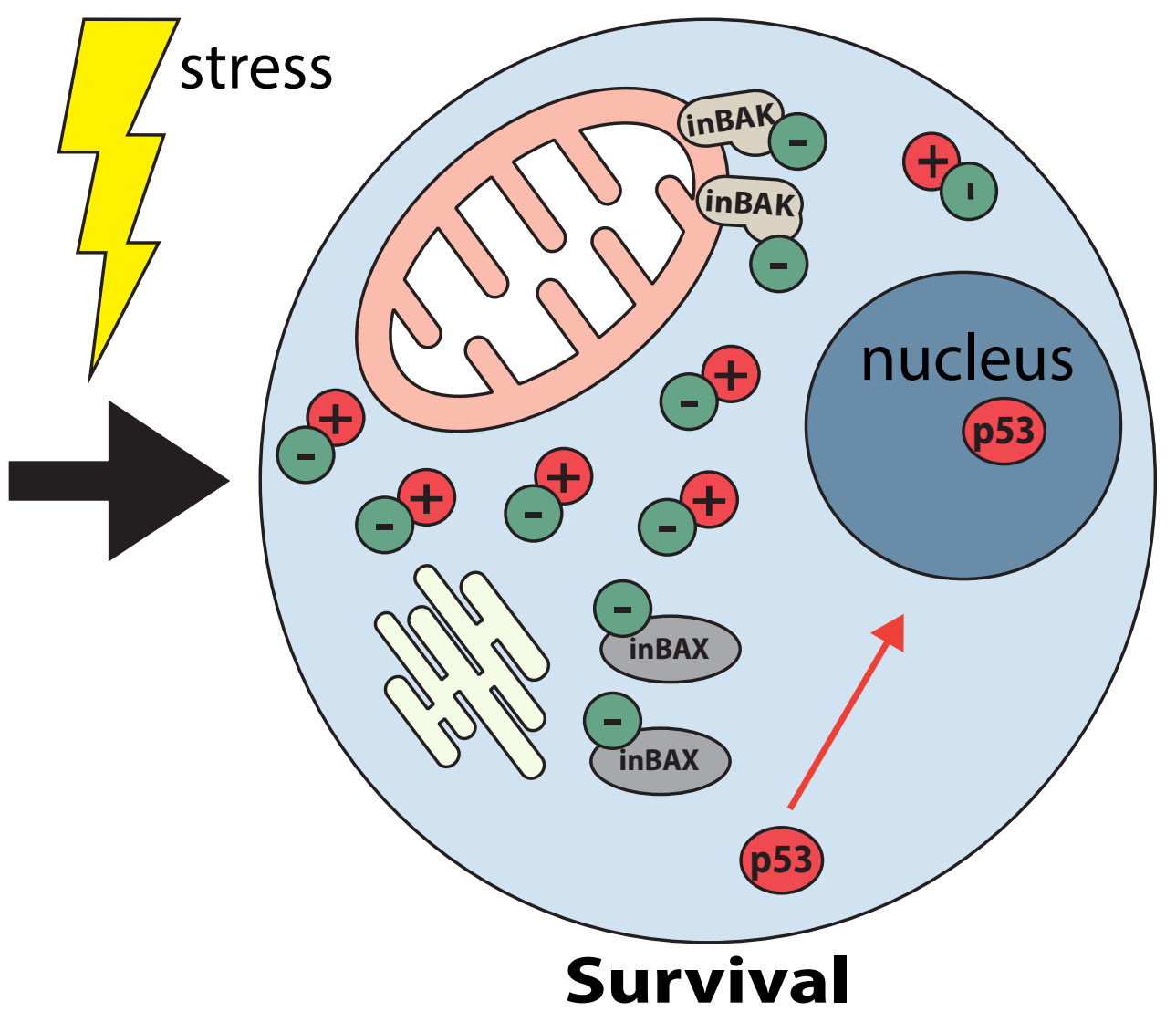


Figure 2
glucose

Figure 2
glucose

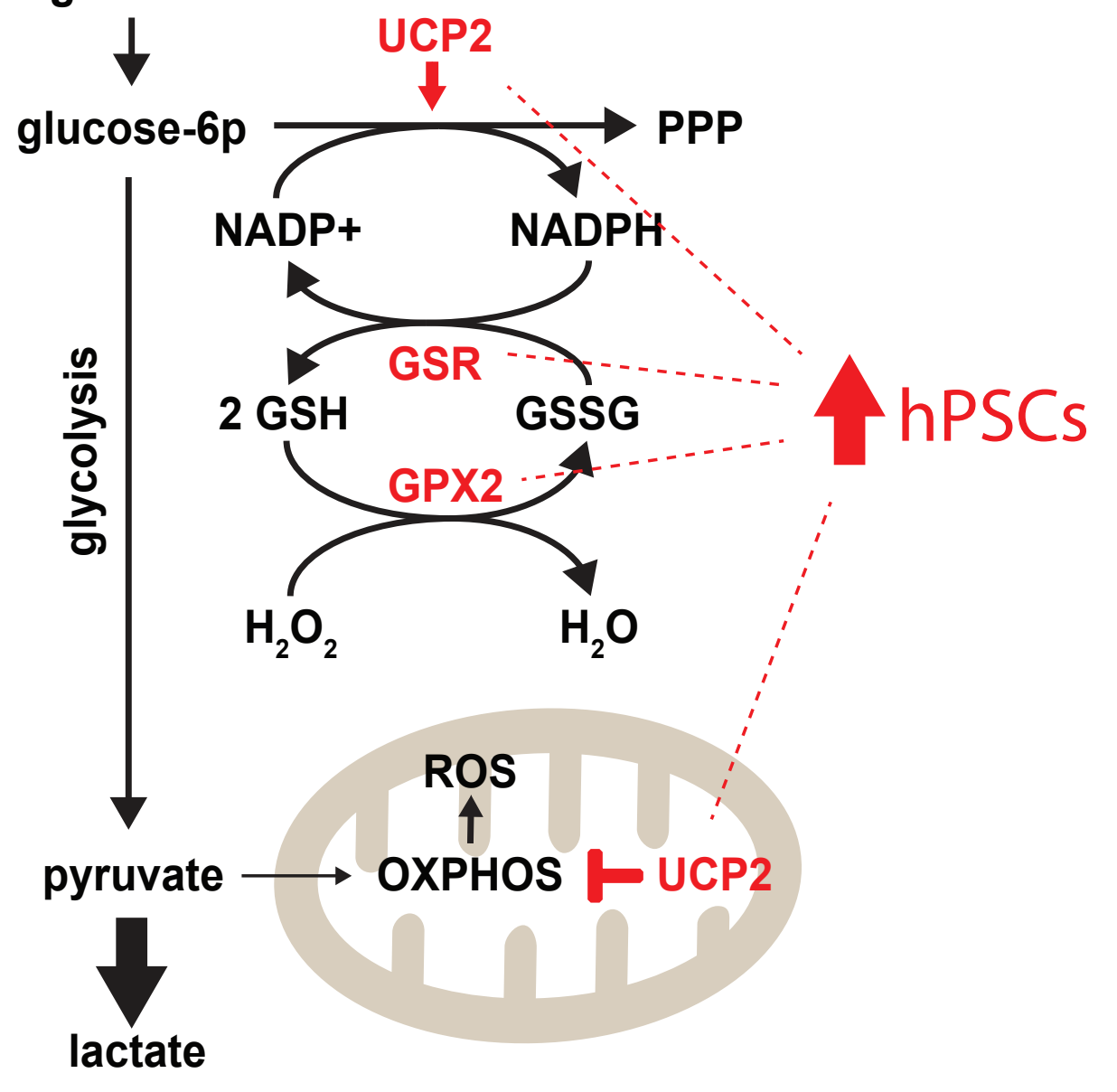

